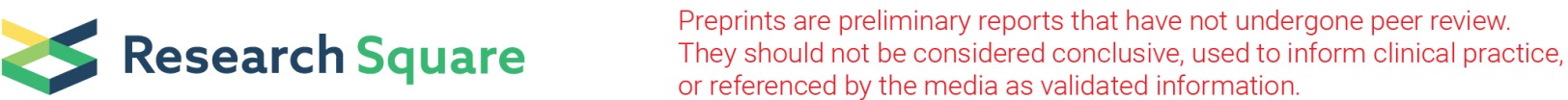

\section{To Identify Risk Factors Predicting Spontaneous Preterm Labor Less Than 28 Weeks After Emergency Cerclage in the Second Trimester of Pregnancy}

\section{Zhucan}

department of obstetrics and gynecology, henan provincial prople's hospital

Yu Ning (D936888523@qq.com )

department of obstetrics and gynecology, henan provincial prople's hospital

Wu Haiying

department of obstetrics and gynecology, henan provincial prople's hospital

\section{Research Article}

Keywords: Prolapsed membranes, Emergency cerclage, spontaneous preterm birth, histologic chorioamnionitis

Posted Date: December 9th, 2021

DOI: https://doi.org/10.21203/rs.3.rs-1078491/v1

License: (a) (1) This work is licensed under a Creative Commons Attribution 4.0 International License.

Read Full License 


\section{Abstract}

Purpose To identify risk factors predicting spontaneous preterm labor (sPTB) less than 28 weeks after emergency cerclage in the second trimester of pregnancy.

Methods 106 cases with Prolapsed membranes in the second trimester of pregnancy who underwent emergency cerclage were collected from July 2019-July 2021.39 cases who happened SPTB less than 28 weeks were in case group. 67 cases were in control group.Univariate analysis and logistic regression analysis were used to identify the risk factors for SPTB less than 28 weeks after emergency cerclage.

Results Among the 106 cases,37cases developed sPTB less than 28 weeks, and the incidence was $36.8 \%$, Univariate analysis showed that positive cervical culture,cervical dilatation,the location of amniotic membranes and histologic chorioamnionitis were related with $\mathrm{SPTB}(P<0.05)$. Logistic regression analysis showed that histologic chorioamnionitis was the risk factors for SPTB after emergency cerclage in patients with Prolapsed membranes in the second trimester of pregnancy[relative risk14.603,95\% confidence interval $(\mathrm{Cl}) 2.621-81.369, \mathrm{P}<0.05]$. Amniotic membrane is an independent risk factor for less than 28 weeks of sPTB after emergency cerclage in middle pregnancy [relative risk $11.993,95 \%$ confidence interval(Cl)3.825-37.607,P<0.05].

Conclusion The independent risk factors of SPTB less than 28 weeks after emergency cerclage with prolapsed membranes in the second trimester of pregnancy was histologic chorioamnionitis and Amniotic membrane.

\section{Introduction}

Prolapsed membranes in the second trimester of pregnancy was an obstetric emergency disease.The patient had a painless cervical dilation, which is founded on speculum examination and transvaginal ultrasound. If the effective measures is not taken,a spontaneous abortion within a week.Surgery is a key treatment for prolonging the delivery interval. A emergency cerclage may be insered when the patient presents with a cervix that is already dilated with the membranes bulging into the vaginal. but the pregnant women at high risk of premature rupture of fetal membranes and spontaneous preterm birth after postoperative.Spontaneous preterm delivery of less than 28 weeks is the most common serious complication after emergency cervical cerclage. premature babies are at greater risk of cerebal palsy,visual and auditory disturbances[1].Preterm birth remains the major cause of mortality and morbidity before and after birth,create a huge public health burden[2].There are still no specific indicators to predict the occurrence of SPTB. Therefore, the risk factors for SPTB occurrence in patients after emergency cerclage were analyzed,It is of great significance to discuss the prevention and control measures,This paper includes 106 cases admitted to our hospital from July 2019 to July 2021 in a retrospective study,which of inserting an emergency cerclage, The occurrence and risk factors of s PTB after less than 28 weeks were analyzed. 


\section{Materials And Methods}

This study was based on a single-center and prospective clinical study in patients with spontaneous premature birth after emergency cervical cerclage in Henan Province, which was reviewed and approved by the Ethics Committee of Henan Provincial People's Hospital.The 106 patients in July 2019 to July 2021 were selected in the clinic,The degree of cervical dilation were found by speculum examination and transvaginal ultrasound.

\subsection{Inclusion criteria}

16-26+6 weeks singleton;a painless cervical dilation with 1-6cm;bulging of the membranes into the vagina,the membranes was intact;at least one second trimester pregnancy loss, or preterm birth;no contractions, normal body temperature, and no uterine tenderness.

\subsection{Exclusion criteria}

a painless cervical dilation $>7 \mathrm{~cm}$;premature rupture of fetal membranes;active vaginal bleeding;the fetal structure was abnormal.

\section{3 surgical operation}

Cervical cerclage was performed using the McDonald technique: the trendelenbury posititon, epidural anesthesia was used.Two double ten sutures stitched into the anterior cervical lip,left wall, right wall, and post-erior cervical lip.The suture should be deep when stitching the anterior cervical lip,Suture enough cervical interstima, which is in 1/3-2/3 cervical thickness,avoid sliding, but do not sew into the cervical canal,Small cervical matrix suture should be stitched on the cervical side wall,Fixed braid line in place,Avoid hurting the uterine arteries, The line tail is knotted in the front,Keep $3-4 \mathrm{~cm}$ for subsequent removal.Cervical cerclage is usually stitched into the needle and needle point, respectively at 1-11 points, 10-8 points, 7-5 points, 4-2 points, up and down two stitches and right two stitches are basically parallel. All patients were treated by the same group of senior physicians using the standard McDonald suture technique.

If the protrusion of the fetal membranes still in the cervical canal,Self-made balloon push amniotic sac technology is used.The tip of the catheter air bag was cut off and the sterile middle finger glove of the coat was used to prevent the puncture of the tip of the catheter. The balloon was injected with $10-30 \mathrm{ml}$ of saline and draining a prolapsed fetal membranes return into the uterine cavity,After the suture, the saline was removed, and the catheter and sterile glove skin were removed. If the saline was $10-30 \mathrm{~mL}$, the sterile glove skin was intact without damaged, which confirmed that no suture penetrated the cervical stroma during surgery. If the amniotic sac expands out to the cervical cervix, use wet gauze to push the amniotic sac to the cervical cervix, tighten the suture after suture, and gently exit the wet gauze. 


\section{4 postoperative management of emergency cerclage}

If there are no complications after cerclage, he will be in hospital 7-14 days after cerclage,Peripheral insurance is performed every 2 weeks to monitor the length of the cervical length and fetal development. For the occurrence of regular uterine contraction after cervical cerclage, premature birth or late abortion is inevitable, timely removal of cervical line, The placenta was sent for pathological examination.

\subsection{Pregnancy outcomes}

The primary outcomes were the rate of SPTB $<28$ weeks and to identify the risk factors for SPTB $<28$ weeks after emergency cerclage.

The secondary outcome were delivery interval, pregnancy at delivery, neonatal birth weight, respiratory distress syndrome of newborn, neonatal hospital stays and placental histological chorioamnionitis.

\subsection{Statistical analysis}

Data were processed with SPSS25.0, quantitative data meeting the normal distribution using mean standard deviation, two independent sample t-tests, median M for non-normal distribution (P25, P75) and non-parametric test Mann-whitney $U$ test. Qualitative data are expressed as frequency and percentage,used c2 tests or continuous correction c2 tests compared between less than the 28-week SPTB and non-SPTB groups.Statistically significant variables were included in the Logistic regression analysis, $P<0.05$ was considered as a statistically significant difference.

\section{Results}

2.1 Table 1 shows Patient demographic characteristics and general basic clinical characteristics comparing case group and control group.Despite emergency cerclage in 106 cases, $39 / 106(36.8 \%)$ delivered<28weeks' gestation. Delivery weeks( $27(26,28)$ vs $31(29,36), P=0.000)$. There were significant differences between delivery interval weeks, Inside cervix expansion,outside cervix expansion,residual cervical length,neonatal birth weigh,neonatal hospital stays compared with control group $(P<0.05)$.There were no significant differences between age and gestational weeks compared with control group $(P>0.05)$.

2.2 Table 2 shows univariate analysis of preterm birth at less than 28 weeks.Cervical dilation at diagnosis(relative risk10.629,95\% confidence interval(Cl)3.511-32.17,P<0.001). Amniotic membrane(relative risk11.508,95\% confidence interval(CI)4.407-30.048,P<0.001), Positive cervical culture(relative risk4.197, 95\% confidence interval(Cl)1.552-11.354,P<0.001). Histological chorioamnionitis $(\mathrm{HCA})$ (relative risk12.956,95\% confidence interval $(\mathrm{Cl}) 4.001-48.579, \mathrm{P}<0.001)$. 
There were statistical significant differences in cervical dilation at diagnosis, amniotic membrane, positive cervical culture,and histological chorioamnionitis(HCA) compared with control group $(P<0.05)$. There were no statistical significant differences between gestational age $(G A)$ at diagnosis, embryo transplantation, cervix operation, and chronic hypertension and diabetes mellitus compared with the control group $(P>0.05)$.

2.3 Table 3 shows results of a Logistic regression analysis of less than 28 weeks of preterm birth,Whether it occurred for less than 28 weeks of SPTB was used as the dependent variable,Logistic regression was performed using statistically significant factors in univariate analysis as independent variables, The results shows histologic chorioamnionitis is an independent risk factor for less than 28 weeks of SPTB after emergency cerclage in middle pregnancy (relative risk14.603,95\% confidence interval $(\mathrm{Cl}) 2.621-81.369, \mathrm{P}<0.05)$. The results shows Amniotic membrane is an independent risk factor for less than 28 weeks of SPTB after emergency cerclage in middle pregnancy (relative risk11.993,95\% confidence interval(Cl)3.825-37.607,P<0.05).

2.4 Table 4 shows neonatal outcome. There were statistical significant differences in severe neonatal asphyxia, hyperbilirubinemia, respiratory distress syndrome, premature fetal membrane rupture $(P R O M)$ compared with control group $(P<0.05)$. There were no statistical significant differences between assisted breath,and neonatal Intensive care unit(NICU)compared with the control group $(P>0.05)$.

Table 1 Basic characteristics of the patients in both patient groups 


\begin{tabular}{|c|c|c|c|}
\hline Variable & $\begin{array}{l}\text { Case group } \\
(n=39)\end{array}$ & $\begin{array}{l}\text { Control group } \\
(n=67)\end{array}$ & $P$ \\
\hline Age(years) & $29.10 \pm 0.63$ & $30.25 \pm 0.47$ & 0.080 \\
\hline Gestational weeks(weeks) & $20.99 \pm 0.57$ & $20.07 \pm 0.47$ & 0.859 \\
\hline \multicolumn{4}{|l|}{ Delivery weeks(weeks) } \\
\hline$M(P 25 \bigotimes P 75)$ & $27 \rrbracket 26,28 \rrbracket$ & $31(29,36)$ & 0.000 \\
\hline \multicolumn{4}{|l|}{ Delivery interval(weeks) } \\
\hline$M(P 25 \bigotimes P 75)$ & $4.43(3.43,7.00)$ & $12.57(7,15.43)$ & 0.000 \\
\hline \multicolumn{4}{|l|}{ Inside cervix expansion $\ \mathrm{~mm} \rrbracket$} \\
\hline$M(P 25 \bigotimes P 75)$ & $28 \rrbracket 20,39 \rrbracket$ & $18 \bigotimes 12,21 \rrbracket$ & 0.001 \\
\hline \multicolumn{4}{|c|}{ Outside cervix expansion(mm) } \\
\hline$M(P 25 \bigotimes P 75)$ & 16ه7,30》 & $8.5 \rrbracket 1,15 \rrbracket$ & 0.001 \\
\hline \multicolumn{4}{|l|}{ Residual cervical length $(\mathrm{mm})$} \\
\hline$M(P 25 \bigotimes P 75)$ & $8.9(1,12)$ & $18(10,21)$ & 0.000 \\
\hline Neonatal birth weight $(\mathrm{g})$ & $909.36 \pm 41.22$ & $1999.33 \pm 110.59$ & 0.000 \\
\hline \multicolumn{4}{|c|}{ Neonatal hospital stays(days) } \\
\hline$M(25 \bowtie P 75)$ & $45(28,53)$ & $29(7,39)$ & 0.000 \\
\hline
\end{tabular}

Table 2 A univariate analysis with less than 28 weeks of SPTB 


\begin{tabular}{|c|c|c|c|c|c|c|}
\hline Variable & $\begin{array}{l}\text { Case } \\
\text { group } \\
(n=39)\end{array}$ & $\begin{array}{l}\text { Control } \\
\text { group } \\
(n=67)\end{array}$ & $c^{2}$ & $P$ & $O R$ & $\begin{array}{l}95 \% \quad \mathrm{Cl} \\
\text { 眈 }\end{array}$ \\
\hline \multicolumn{7}{|l|}{ GA at diagnosis } \\
\hline$\leq 22$ weeks & $20 \otimes 51.3 \rrbracket$ & $32(47.8)$ & 0.122 & 0.727 & 0.869 & $\begin{array}{r}0.394 \\
1.914\end{array}$ \\
\hline$>22$ weeks & 19ه48.7区 & $35 \rrbracket 52.2 \rrbracket$ & & & & \\
\hline \multicolumn{7}{|l|}{$\begin{array}{l}\text { Cervical dilation at } \\
\text { diagnosis }\end{array}$} \\
\hline$\leq 4 \mathrm{~cm}$ & $21 \otimes 53.8 \rrbracket$ & $62 \rrbracket 92.5 \rrbracket$ & 21.720 & 0.000 & 10.629 & $\begin{array}{l}3.511 \\
32.17\end{array}$ \\
\hline$>4 \mathrm{~cm}$ & $18 \rrbracket 46.2 \rrbracket$ & $5 \otimes 7.5 \rrbracket$ & & & & \\
\hline \multicolumn{7}{|l|}{ Amniotic membrane } \\
\hline within cervical canal & $14 \llbracket 35.9 \rrbracket$ & $58 \rrbracket 86.6 \rrbracket$ & 29.049 & 0.000 & 11.508 & $\begin{array}{l}4.407 \\
30.048\end{array}$ \\
\hline Beyond external os & $25 \rrbracket 64.1 \rrbracket$ & $9 \rrbracket 13.4 \rrbracket$ & & & & \\
\hline $\begin{array}{l}\text { Cervical V-shaped } \\
\text { expansion }\end{array}$ & $21 \otimes 53.8 \rrbracket$ & $18 \rrbracket 56.7 \rrbracket$ & 4.275 & 0.039 & 0.427 & $\begin{array}{l}0.189 \\
0.964\end{array}$ \\
\hline embryo transplantation & $24 \llbracket 61.5 \rrbracket$ & $46 \rrbracket 68.7 \rrbracket$ & 0.557 & 0.456 & 1.369 & $\begin{array}{l}0.599 \\
3.128\end{array}$ \\
\hline Positive cervical culture & $33 \llbracket 84.6 \rrbracket$ & $38 \rrbracket 56.7 \rrbracket$ & 8.676 & 0.003 & 4.197 & $\begin{array}{l}1.552 \\
11.354\end{array}$ \\
\hline Secondary cerclage & $7 \rrbracket 17.9 \rrbracket$ & $2 \varangle 3.0 \otimes$ & 5.309 & 0.021 & 7.109 & $\begin{array}{l}1.396 \\
36.194\end{array}$ \\
\hline $\mathrm{HCA}$ & $37 ه 94.9 \rrbracket$ & $34 \rrbracket 50.7 \rrbracket$ & 21.702 & 0.000 & 12.956 & $\begin{array}{l}4.001 \\
48.579\end{array}$ \\
\hline Cervix operation & $3 \otimes 7.7 \rrbracket$ & $5 \otimes 7.5 \rrbracket$ & 0.004 & 0.951 & 0.670 & $\begin{array}{l}0.124 \\
3.631\end{array}$ \\
\hline Chronic hypertension & $3 \otimes 7.7 \rrbracket$ & $2 \varangle 3.0 \otimes$ & 0.394 & 0.530 & 2.708 & $\begin{array}{l}0.432 \\
16.967\end{array}$ \\
\hline Diabetes mellitus & $10 \rrbracket 25.6 \rrbracket$ & $15 \rrbracket 22.4 \rrbracket$ & 0.145 & 0.704 & 1.195 & $\begin{array}{l}0.476 \\
3.000\end{array}$ \\
\hline
\end{tabular}

Table 3 Results of the Logistic regression analysis 


\begin{tabular}{|llllll|}
\hline Variable & $\mathrm{b}$ & $\mathrm{SE}$ & Wald c$^{2}$ & $P$ & $O R \otimes 95 \% \mathrm{Cl}$ \\
\hline Positive cervical culture & 0.627 & 0.667 & 0.884 & 0.347 & $1.873 \llbracket 0.506-6.924 \rrbracket$ \\
\hline Cervical dilation at diagnosis & 0.866 & 0.558 & 2.407 & 0.121 & $0.421 \rrbracket 0.141-1.256 \rrbracket$ \\
\hline Amniotic membrane & 2.484 & 0.583 & 18.152 & 0.000 & $11.993 \rrbracket 3.825-37.607 \rrbracket$ \\
\hline HCA & 2.681 & 0.876 & 9.359 & 0.002 & $14.603 \rrbracket 2.621-81.369 \rrbracket$ \\
\hline
\end{tabular}

Table 4 Neonatal pregnancy outcomes in both groups

\begin{tabular}{|c|c|c|c|c|c|c|}
\hline \multirow[t]{2}{*}{ Variable } & \multirow{2}{*}{$\begin{array}{l}\text { Case } \\
\text { group } \\
(n=39)\end{array}$} & \multirow{2}{*}{$\begin{array}{l}\text { Control } \\
\text { group } \\
(n=67)\end{array}$} & \multirow[b]{2}{*}{$c^{2}$} & \multirow{2}{*}{$P$} & \multirow{2}{*}{ OR } & \multirow{2}{*}{$95 \% \mathrm{Cl}$} \\
\hline & & & & & & \\
\hline Vaginal delivery & $31 \otimes 79.5 \rrbracket$ & $33 \rrbracket 49.3 \rrbracket$ & 9.419 & 0.002 & 0.250 & $\begin{array}{l}0.101 \\
0.624\end{array}$ \\
\hline Severe neonatal asphyxia & $15(38.5)$ & $2(3.0)$ & 20.481 & 0.000 & 20.313 & $\begin{array}{l}4.230 \\
95.501\end{array}$ \\
\hline Hyperbilirubinemia & $16 \rrbracket 41.0 \rrbracket$ & 11(16.4) & 7.863 & 0.005 & 3.542 & $\begin{array}{l}1.428 \\
8.783\end{array}$ \\
\hline $\begin{array}{l}\text { Respiratory distress } \\
\text { syndrome }\end{array}$ & $31(79.5)$ & $30 \bigotimes 44.8 \rrbracket$ & 12.157 & 0.000 & 4.779 & $\begin{array}{l}1.915 \\
11.924\end{array}$ \\
\hline Trachea cannula & $11 \rrbracket 28.2 \bigotimes$ & $2 \varangle 3.0 \rrbracket$ & 12.322 & 0.000 & 12.768 & $\begin{array}{l}2.655 \\
61.397\end{array}$ \\
\hline Assisted breath & $21 \otimes 53.8 \rrbracket$ & $32 \rrbracket 47.8 \rrbracket$ & 0.365 & 0.546 & 1.276 & $\begin{array}{l}0.578 \\
2.815\end{array}$ \\
\hline $\mathrm{NICU}$ & $34 \llbracket 87.2 \rrbracket$ & 48\71.6ه & 3.398 & 0.065 & 2.692 & $\begin{array}{l}0.915 \\
7.915\end{array}$ \\
\hline PROM & $30 \rrbracket 76.9 \rrbracket$ & $24 \rrbracket 35.8 \rrbracket$ & 16.664 & 0.000 & 5.972 & $\begin{array}{l}2.436 \\
14.643\end{array}$ \\
\hline
\end{tabular}

GA,gestational age囚PROM囚premature rupture of membranes;NICU,Neonatal Intensive Care Unit;HCA,histological chorioamnionitis; OR,odds ratio; $\mathrm{Cl}$, confedence interval

\section{Discussion}

3.1 Treatment effect of emergency cervical cerclage on Prolapsed membranes in the second pregnancy Cervical insufficiency is the main cause of painless progressive cervical dilation in the second pregnancy and extremely preterm birth[3].Cervical cerclage represents a surgical intervention to prevent spontaneous 
preterm birth,Emergency cervical cerclage reduces preterm birth, provides prolonged gestational weeks, and reduces neonatal mortality and loss rates. SOGC[4] recommends emergency cervical cerclage in a singleton pregnant cervix less than $4 \mathrm{~cm}$ without contractions. The Han et al [5] retrospective study analyzed the prolonged pregnancy interval of 5-7 weeks after cervical dilation $>1 \mathrm{~cm}$ compared to the conservative treated group.Emergency cervical cerclage is an active treatment that extends gestational weeks and increases neonatal survival, However,premature fetal membrane rupture occurs in 4\%-9\% postoperative cases, when the amniotic cavity intrauterine infection is present, the effect of cerclage decreases significantly[6].Several studies have shown that the amniotic sac is located beyond external os and that the cervix dilation of $1.5 \mathrm{~cm}$ is an independent risk factor for failure after cerclage[7],The Baryk M et al[8] study evaluated emergency cervical cerclage with less than 28 weeks of premature birth at 75/252 (29.7\%),In this study, 106 patients underwent emergency cervical dilation and amniotic protrusion, and 39 had SPTB less than 28 weeks with an incidence of $36.8 \%$,Higher than the above study may be related to a higher proportion of cervical expansion greater than $4 \mathrm{~cm}$ (18 cases). Emergency cervical cerclage has a positive effect on pregnancy outcome with cervical dilation $>4 \mathrm{~cm}$, less than spontaneous prematurity of $18 / 37(46.2 \%)$ at 28 weeks, prolonged gestational interval of $4.43(3.43,7.00)$ weeks. In patients with cervical dilation $>4 \mathrm{~cm}$ and prolapsed membrane emergency cerclage is an intervention to extend the gestational week to reach the viable ability of the fetus. To avoid this emergency, predicting risk factors for spontaneous preterm birth is an important part of current prenatal clinical work[9]. We advocate midtrimester ultrasound for cervical length screening and is a predictor of the prevention of sPTB[10].Cervical insufficiency is painless, lack of obvious clinical symptoms, no objective diagnosis, and is largely dependent on clinical experience, production history, and retrospective diagnosis of ultrasound scan.Through color ultrasound examination, the degree of cervical internal and external expansion, V-shaped,U-shaped expansion, the length of cervical closure section, to guide clinical intervention measures as soon as possible.In this study, the degree of cervical dilation is greater than $4 \mathrm{~cm}$, and less than 28 weeks of spontaneous prematurity (case group) of 18/39 (46.2\%) is signi-ficantly higher than that in the control group of $5 / 67$ (7.5\%), The amniotic sac is beyond external os $25 / 39(64.1 \%)$ higher than in control group 9/67 (13.4\%).In the second trimester of pregnancy, color ultrasound is used to find the cervical opening situation and the location of the amniotic membrane,lt is of great clinical significance to guide early clinical interventions to reduce spontaneous premature birth.

3.2 Analysis of risk factors for spontaneous preterm birth at less than 28 weeks after emergency cerclage.

Emergency cerclage is a rescue procedure, but there are still some premature deliveries after surgery, and the biological mechanism is not completely clear. Preterm birth in China is defined as delivery between 28 and 37 weeks, so 28 weeks gestation was chosen as the cutoff for the study factors associated with the clinical effect of cervical cerclage.Extreme preterm birth is defined as delivery at less than 28 weeks of gestation[11].The etiology of extreme preterm birth is mainly divided into two categories: intrauterine infection / inflammation and placental vascular dysfunction,The first type of etiology is associated with chorioamnionitis, premature fetal membrane rupture, and cervical insufficiency, The second etiology is associated with hypertension during pregnancy and fetal growth restriction[12].Alfirevic et a[13]An 
increased risk of chorioamnionitis in patients with cervical insufficiency is systematically reported. Chorioamnionitis (chorionamnionitis,CAM), an inflammatory response formed by placental tissue by pathogen infection, is a common complication during pregnancy, divided into clinical chorioamnionitis (clinical chorioamnionitis,CCA) and tissue chorioamnionitis (histological chorioamnionitis, HCA),HCA refers to no clinical manifestations only on postnatal pathological examination, 2-3-fold incidence of CCA[14].HCA is considered to be the major cause of extreme preterm delivery, and a substantial metaanalysis of chorioamnionitis is strongly associated with adverse pregnancy outcomes in extreme preterm birth with complications such as bronchopulmonary dysplasia[15], Premature retinopathy of the disease[16], neonatal sepsis, and respiratory distress syndrome[17].The Uzun Cilingir et al[18]study found that the incidence of chorioamnionitis after emergency cervical cerclage is $22 / 44$ (50\%).this study shows that tissue chorionic amnionitis is an independent risk factor for SPTB less than 28 weeks after emergency cervical cerclage of prolapsed membranes in second pregnancy,Postintrapartum placental tissue chorioamnionitis less than 28 weeks (case group) was 37/39 (94.9) significantly higher than control 34/67 (50.7\%),This study divided the delivery week into groups at 28 weeks, the smaller the delivery week, the higher the incidence of chorionic amnionitis, less than 28 weeks of spontaneous preterm birth (case group) neonatal respiratory distress syndrome $31 / 39(79.5 \%)$ than the control group $30 / 67$ (44.8\%), which is basically in agreement with the above report.

In conclusion, In patients with cervical dilation and prolapsed membranes, emergency cerclage is an important intervention to prolong the gestational week of delivery, with a postoperative incidence of SPTB less than 28 weeks of $36.8 \%$, Tissue chorioamnionitis and amniotic membrane are independent risk factor for less than 28 weeks of SPTB after emergency cerclage in middle pregnancy.In this study, it identifies risk factors affecting spontaneous preterm birth at less than 28 weeks after emergency cerclage. Moreover, timely and effective intervention in patients after cervical cerclage to reduce spontaneous preterm birth of less than 28 weeks, Maximize the pregnancy week beyond the risk of last pregnancy loss, It is expected to extend the gestational week to reach fetal viability and reduce complications in preterm newborns. Although most confounders are eliminated in this study, some risk factors may be excluded due to the small sample size, especially in the case group,As this study is a single-enter retrospective clinical study, the statistical process is inevitably biased and has certain limitations, and large samples of multicenter studies are still needed in the next step.

\section{Declarations}

Research funding:This work was supported by the Henan 2019 Science and Technology Development Plan(192102310335).

\section{Author contribution:}

Zhucan: protocol, data collection, and manuscript writing.Liuyuning:data management, data analysis.

Wuhaiying:project development and manuscript editing. 
Conflict of interest All authors declare no conficts of interest.

Ethical approval The study has been approved by the Ethics Committee at the Henan Provincial People's Hospital

\section{References}

1. Dabi Y, Nedellec S, Bonneau C, etal. Clinical validation of a model predicting the risk of preterm delivery[J]. PIOS One ,2017;12(2):e0171801.

2. Umeigbo BC, Modebe IA, lloghalu IC, et al. Outcomes of preterm labor and preterm births: a retrospective cross-sectional analytical study in a Nigerian single center population [J]. Obstetrics and Gynecology Research,2020;3(1):17-28.

3. Robertson JE, Lisonkova S, Lee T,et al. Fetal, Infant and Maternal Outcomes among Women with Prolapsed Membranes Admitted before 29 Weeks Gestation [J]. PLoS One,2016;11(12):168-285.

4. Brown R囚Gagnon R囚Delisle MF. No. 373-Cervical Insufficiency and Cervical Cerclage [J]. Obstet Gynaecol Can,2019,41(2):233-247.

5. Han MN, O'Donnell BE, Maykin MM, et al. The impact of cerclage in twin pregnancies on preterm birth rate before 32 weeks [J]. Matern Fetal Neonatal Med,2019;32(13):2143-2151.

6. Monckeberg M,Valdes R,Kusanovic JP, et al. Patients with acute cervical insufficiency without intraamniotic infection /inflammation treated with cerclage have a good prognosis [J].Perinal Med,2019;47(5):500-509.

7. Abu Hashim H囚Al-Inany H囚Kilani Z.A review of the contemporary evidence on rescue cervical cerclage [J].Int J Gynecol Obstet,2014;124(3):198-203.

8. Bayrak M,Gul A,Goynumer G.Rescue cerclage when foetal membranes prolapse into the vagina[J] .Obstet Gynaecol(lahore),2017;37(4):471-475.

9. Asiegbu AC, Eleje GU, Ibeneme EM, et al. Combined insulin-like growth factor binding protein1/interleukin-6 (Premaquick) versus fetal fibronectin for predicting preterm delivery among women with preterm contractions [J]. International Journal of Gynaecology and Obstetrics,2020;149(2):1717.

10. Murray SR, Stock SJ, Cowan S, Cooper ES, Norman JE. Spontaneous preterm birth prevention in multiple pregnancy[J] . The Obstetrician \& Gynaecologist, 2018;20(1);57-63.

11. Moutquin JM. Classifification and heterogeneity of preterm birth[J] . BJOG, 2003;110(Suppl. 20):303.

12. Gagliardi L, Rusconi F, Bellu R, et al. Association of maternal hypertension and chorioamnionitis with preterm outcomes [J]. Pediatrics,2014;134(1):154-161.

13. Alfirevic Z, Stampalija T, Medley N. Cervical stitch (cerclage) for preventing preterm birth in singleton pregnancy[J]. Cochrane Database of Systematic Reviews ,2017(6):CD008991. 
14. Strauss JF,Romero R,Gomez-Lopez N,et al. Spontaneous preterm birth囚advances toward the discovery of genetic predisposition[J]. Am J Obstet Gynecol, 2018,218(3):294-314.

15. Villamor-Martinez E, Fumagalli M, Mohammed Rahim O, et al.Chorioamnionitis is a risk factor for intraventricular hemorrhage in preterm infants: a systematic review and meta-analysis [J]. Front Physiol, 2018;9:1253.

16. Villamor-Martinez E, Cavallaro G, Raffffaeli G, et al. Chorioamnionitis as a risk factor for retinopathy of prematurity: an updated systematic review and meta-analysis [J]. PLoS ONE,2018; 13(10):e0205838.

17. liu C,Sun J,Liu Y,et al.Different exposure levels of fine particulate matter and preterm birth:a metaanalysis based on cohort studies[J].Environ Sci Pollut Res Int,2017,24(22):17976-17984.

18. Uzun Cilingir I,Sayin C,Sutcu H,et al.Does emergency cerclage really work in patients with advanced cervical dilatation囚[J]. Gynecol Obstet Hum Reprod,2019:48:387-390. 